\title{
Prostate Cancer and Prostatic Diseases Best of Asia, 2019: challenges and opportunities
}

\author{
Yao $\mathrm{Zhu}^{1,2} \cdot$ Stephen J. Freedland ${ }^{3,4} \cdot$ Dingwei $\mathrm{Ye}^{1,2}$
}

Received: 9 November 2019 / Accepted: 14 November 2019 / Published online: 6 December 2019

(c) The Author(s), under exclusive licence to Springer Nature Limited 2019

The incidence of prostate cancer (PCa) varies widely among different ethnicities, while 73.7 per 100,000 in North America and 13.9 per 100,000 in East Asia [1]. Although the incidence of $\mathrm{PCa}$ in the East Asian population is relatively low, it has grown rapidly in recent years, owing to increased life expectancy and westernization of lifestyle. Nowadays, more than 297,000 new PCa patients are diagnosed in Asia annually, accounting for $23.3 \%$ globally (Global Cancer Observatory: http://gco.iarc.fr/). Moreover, $\sim 33 \%$ new deaths occurred in Asia (more than 118,000 patients). With the rapid development of Asian urology in recent years, especially the increasing quality and quantity of PCa clinical trials in East Asian population, more and more high-quality papers from Asia have been published in many high-impact journals [2]. Thus, the international academic community is paying more and more attention to urological diseases, especially prostatic diseases in Asia. With the deepening of research, similarities and disparities of prostatic disease patients (especially prostate cancer patients) between Asian and Western populations have been elucidated gradually, from clinical features to genetics and genomics. Editor-in-chief (SJF) and editorial board members of Prostate Cancer and Prostatic Diseases have a great interest in prostate cancer and other prostatic diseases' spectrum in Asia, and try to attract more attention in

$\triangle$ Dingwei Ye

dwye.shca@gmail.com

1 Department of Urology, Fudan University Shanghai Cancer Center, Shanghai 200032, China

2 Department of Oncology, Shanghai Medical College, Fudan University, Shanghai, China

3 Department of Surgery, Division of Urology, Samuel Oschin Comprehensive Cancer Institute, Cedars-Sinai Medical Center, Los Angeles, CA, USA

4 Surgery Section, The Durham VA Medical Center, Durham, NC, USA collaborative studies across ethnicities. In recognition of this, during the 9th Shanghai Genitourinary Oncology International Symposium (29th Nov-1st Dec, 2019), the editorial team of Prostate Cancer and Prostatic Diseases will present "Best of Asia 2019 Issue" in Shanghai, China. This is a fantastic opportunity to introduce Asia's achievements in prostate research and promote collaboration.

This year's Prostate Cancer and Prostatic Diseases "Best of Asia" issue is composed of eight papers written by Asian urologists. They are as follows:

1. Therapeutic potential of ReACp53 targeting mutant p53 protein in CRPC [3]. This research indicates a potential role of $\mathrm{ReACp} 53$ as a single therapeutic regimen that could be applied to the treatment of advanced prostate cancer.

2. The role of Prostate Imaging Reporting and Data System score in Gleason $3+3$ active surveillance candidates enrollment: a diagnostic meta-analysis [4]. This meta-analysis shows that patients with Gleason Score $3+3$ but PI-RADS 4-5 should not receive active surveillance even though they fulfill current criteria for active surveillance.

3. Prediction of extraprostatic extension by MRI tumor contact length: difference between anterior and posterior prostate cancer [5]. This paper indicates that anterior tumors have more favorable pathological characteristics than posterior tumors with the same extent of contact between prostate cancer and the prostatic capsule measured by MRI.

4. Efficacy and safety of periprostatic nerve block combined with perineal subcutaneous anaesthesia and intrarectal lidocaine gel in transrectal ultrasound guided transperineal prostate biopsy: a Prospective Randomised Controlled Trial [6]. Authors indicate that periprostatic nerve block combined with subcutaneous perineal anaesthesia and intrarectal lidocaine gel is an efficient approach to local anaesthesia for transperineal prostate biopsy, and is worthwhile applying in the clinic. 
5. Androgen-deprivation therapy for prostate cancer and the risk of autoimmune diseases [7]. This article shows that ADT use in patients with PCa is associated with a decreased risk of autoimmune diseases, which provides a potential role for androgen-deprivation therapy in the modification of inflammation and autoimmunity in Asian PCa patients.

6. Importance of androgen-deprivation therapy during enzalutamide treatment in men with metastatic castration-resistant prostate cancer following chemotherapy: results from retrospective, multicenter data [8]. Authors find concomitant ADT during enzalutamide treatment may improve the survival of patients with mCRPC following Chemotherapy.

7. Preoperative PI-RADS Version 2 scores helps improve accuracy of clinical nomograms for predicting pelvic lymph node metastasis at radical prostatectomy [9]. Researchers confirm the usefulness of preoperative PIRADSv2 scores in helping predict positive pelvic lymph node metastasis at radical prostatectomy.

8. Efficacy and safety of enucleation vs. resection of prostate for treatment of benign prostatic hyperplasia: a meta-analysis of randomized controlled trials [10]. Authors indicate that enucleation has better efficacy and safety postoperatively with less hematological changes and severe complications, compared with resection in treating benign prostatic hyperplasia.

As high-quality researches from Asia increases, we have found more and more clinical differences, such as ERGfusion positive rate [11] and response to androgendeprivation treatment [12], between prostate cancer patients in Asia and Europe. Although the development of urology in Asia is rapid, there is still a gap compared with Europe and the United States. Due to differences in race/ ethnicity and lack of large-scale clinical trial data based on Asian populations, whether existing popular clinical guidelines (EAU/AUA/NCCN) can be directly used in Asian populations is still debated. Thus, in the era of precision medicine and evidence-based medicine, we should not only identify the differences between ethnicities, but also explore why the differences exist and how to implement the ideal treatment for Asian patients. So, in this year's Shanghai Genitourinary Oncology International Symposium, prominent professors from Asia will share data and opinions, and intend to reach a consensus to improve management of prostate cancer patients in Asia. We hope that this Best of Asia Issue and the consensus can arouse international interest in prostate cancer in Asia, and greatly promote more large-scale inter-regional studies.

\section{Compliance with ethical standards}

Conflict of interest The authors declare that they have no conflict of interest.

Publisher's note Springer Nature remains neutral with regard to jurisdictional claims in published maps and institutional affiliations.

\section{References}

1. Bray F, Ferlay J, Soerjomataram I, Siegel RL, Torre LA, Jemal A. Global cancer statistics 2018: GLOBOCAN estimates of incidence and mortality worldwide for 36 cancers in 185 countries. CA: Cancer J Clin. 2018;68:394-424.

2. Li N, Huang HY, Wu DW, Yang ZM, Wang J, Wang JS, et al. Changes in clinical trials of cancer drugs in mainland China over the decade 2009-18: a systematic review. Lancet Oncol. 2019;20: e619-26.

3. Zhang Y, Xu L, Chang Y, Li Y, Butler W, Jin E, et al. Therapeutic potential of ReACp53 targeting mutant p53 protein in CRPC. Prostate Cancer Prostatic Dis. 2019, https://doi.org/10.1038/ s41391-019-0172-z.

4. Zhai L, Fan Y, Meng Y, Feng X, Yu W, Jin J. The role of prostate imaging reporting and data system score in gleason $3+3$ active surveillance candidates enrollment: a diagnostic meta-analysis. Prostate Cancer Prostatic Dis. 2019;22:235-43.

5. Matsumoto K, Akita H, Narita K, Hashiguchi A, Takamatsu K, Takeda $\mathrm{T}$, et al. Prediction of extraprostatic extension by MRI tumor contact length: difference between anterior and posterior prostate cancer. Prostate Cancer Prostatic Dis. 2019;22: 539-45.

6. Lv Z, Jiang H, Hu X, Yang C, Chand H, Tang C, et al. Efficacy and safety of periprostatic nerve block combined with perineal subcutaneous anaesthesia and intrarectal lidocaine gel in transrectal ultrasound guided transperineal prostate biopsy: a prospective randomised controlled trial. Prostate Cancer Prostatic Dis. 2019, https://doi.org/10.1038/s41391-019-0155-0.

7. Liu JM, Yu CP, Chuang HC, Wu CT, Hsu RJ. Androgen deprivation therapy for prostate cancer and the risk of autoimmune diseases. Prostate Cancer Prostatic Dis. 2019;22:475-82.

8. Jeong CW, Kang M, Il Jung S, Kim TH, Park SW, Joung JY, et al. Importance of androgen-deprivation therapy during enzalutamide treatment in men with metastatic castration-resistant prostate cancer following chemotherapy: results from retrospective, multicenter data. Prostate Cancer Prostatic Dis. 2019;22:150-8.

9. Huang C, Song G, Wang H, Lin Z, Wang H, Ji G, et al. Preoperative PI-RADS Version 2 scores helps improve accuracy of clinical nomograms for predicting pelvic lymph node metastasis at radical prostatectomy. Prostate Cancer Prostatic Dis. 2019, https:// doi.org/10.1038/s41391-019-0164-z.

10. Zhang Y, Yuan P, Ma D, Gao X, Wei C, Liu Z, et al. Efficacy and safety of enucleation vs. resection of prostate for treatment of benign prostatic hyperplasia: a meta-analysis of randomized controlled trials. Prostate Cancer Prostatic Dis. 2019;22:493-508.

11. Sedarsky J, Degon M, Srivastava S, Dobi A. Ethnicity and ERG frequency in prostate cancer. Nat Rev Urol. 2018;15:125-31.

12. Bernard B, Muralidhar V, Chen YH, Sridhar SS, Mitchell EP, Pettaway CA, et al. Impact of ethnicity on the outcome of men with metastatic, hormone-sensitive prostate cancer. Cancer. 2017;123:1536-44. 